\title{
Impacto de la sonrisa sobre calidad de vida relacionada con salud bucal en adultos
}

\section{Impact of the smile on oral health-related quality of life in adults}

\author{
Shyrley Díaz-Cárdenas ${ }^{1}$, Lesbia Tirado-Amador ${ }^{2}$, Guillermo Tamayo-Cabeza ${ }^{3}$
}

\begin{abstract}
1. Odontóloga, Magister en Salud Pública, especialista en Salud Familiar y Gerencia en Salud, Facultad de Odontología Universidad de Cartagena. Colombia sdiazc@unicartagena.edu.co 2. Odontóloga, Investigadora de la Facultad de Odontología, Universidad de Cartagena. Colombia. Itiradoa@unicartagena.edu.co

3. Estudiante de Odontología, miembro del semillero de investigación, Grupo GISPOUC. Universidad de Cartagena. Colombia.getc_1@hotmail.com

* Correspondencia autor: Shyrley Díaz Cárdenas Facultad de Odontología, Universidad de Cartagena, Campus de la salud, Barrio Zaragocilla, Cartagena de Indias, Colombia. | TGrupo de Investigación en Salud Pública GISPOUC | E-mail: sdiazc@unicartagena.edu.co

Trabajo recibido el 23/02/2017. Aprobado para su publicación el 23/05/2017
\end{abstract}

\section{RESUMEN}

Objetivo. Describir impacto de la sonrisa sobre calidad de vida relacionada con la salud bucal (CVRSB) en pacientes, Facultad de Odontología, Universidad de Cartagena, Colombia. Material y Métodos. Estudio analítico de corte transversal, en 389 adultos seleccionados por muestreo censal. Se indagó: variables sociodemográficas, la sonrisa evaluada de dos formas: Apariencia Clínica: color y tamaño de dientes, encías y labios, número de dientes, presencia de caries, restos radiculares, uso de prótesis dentales y Satisfacción de la Sonrisa: a través del Smile Perception Impact related Quality of Life (SPIRQoL). También se indagó por la CVRSB con el General Oral Health Assesment Index (GOHAI). Fue realizado análisis univariado y multivariado de Regresión de Poisson con varianza robusta y nivel de confianza del $95 \%$. Resultados. 17,7 \% (IC 95\%: 7,6-13,8) presentó insatisfacción con su sonrisa (SPIRQoL) y el dominio autoestima fue el más comprometido; en el modelo multivariado la procedencia rural impactó de manera negativa sobre la satisfacción de la sonrisa (SPIRQoL) (RR: 2,27, IC 95 \%: 1,4-3,6, p=0,001), igual que el tamaño inadecuado de dientes (RR: 10, IC $95 \%: 3,12-32,1, p=0,000$ ), encías (RR: 2,4, IC $95 \%$ : 1,3-4,3, p=0,004) y labios inadecuados (RR: 2,5, IC $95 \%: 1,5-4,4, p=0,001$ ). La insatisfacción de la sonrisa impactó de manera negativa sobre la CVRSB (GOHAI) (RR: 1,19, IC $95 \%: 1,2-1,4, p=0,000)$. Conclusiones. La insatisfacción de la sonrisa genera impacto negativo sobre CVRSB además de la apariencia clínica inadecuada de dientes, encías y labios y ser de procedencia rural.

PALABRAS CLAVE:

Calidad de vida; Sonrisa; Adulto; Atención odontológica.

Rev. Clin. Periodoncia Implantol. Rehabil. Oral Vol. 11(2); 78-83, 2018.

\section{ABSTRACT}

Objective. To assess the impact of the smile on oral health-related quality of life in adults, Faculty of Dentistry, University of Cartagena, Colombia. Material and Methods. Analytic Crosssectional study in 389 subjects aged between 18 and 81 years using Census sampling. The sociodemographic variables were evaluated and the smile assessed, in two ways: Clinical Appearance: teethcolor and size, gums, lips, number of teeth, presence of caries, root fragments, use of dentures and Smile Satisfaction: through the Smile Perception Impact-related Quality of Life (SPIRQoL). Besides, the Oral health-related quality of life (OHRQoL) was evaluated through the General Oral Health Assessment Index (GOHAl). Univariate statistical analysis and multivariate analysis with Poisson regression models were used to associate the different clinical and sociodemographic factors with the outcome. Results. $17,7 \%(\mathrm{Cl} 95 \%=7,6-13,8)$ showed dissatisfaction with their smile (SPIRQoL); the self-esteem was the most compromised domain; in the multivariate model, the rural origin had a negative impact on the smile perception measured by SPIRQoL (RR: 2,27; Cl $95 \%=1,4-3,6 ; p=0,001$ ), just like inadequate size teeth (RR: 10; $\mathrm{Cl} 95$ $\%=3,12-32,1, p=0.000$ ), inadequate gums (RR: $2.4 ; \mathrm{Cl} 95 \%=1,3-4,3 ; p=0,004)$ and inadequate lips (RR: 2,$5 ; \mathrm{Cl} 95 \%=1,5-4,4 ; p=0,001)$. Smile dissatisfaction showed negative impacts on the OHRQoL measured with GOHAI (RR: 1,19; IC 95\%=1,2-1,4; $p=0,000$ ). Conclusion. Smile dissatisfaction showed negative impacts on OHRQoL, in addition to the clinical inadequate appearance of teeth, gums and lips and being from rural origin.

\section{KEYWORDS:}

Quality of Life; Smiling: Adult: Dental Care.

Rev. Clin. Periodoncia Implantol. Rehabil. Oral Vol. 11(2); 78-83, 2018. 


\section{INTRODUCCIÓN}

La salud general se encuentra influenciada por diversos factores y ha sido definida por la Organización Mundial de la Salud como "un completo estado de bienestar físico, mental y social y no solamente la ausencia de enfermedad", por lo que el concepto de salud posee un enfoque multidimensional que abarca síntomas relacionados con funcionamiento físico y el bienestar emocional ${ }^{(1)}$. Este concepto global de salud, el componente de salud bucal también influye en el bienestar general del individuo, al ser la salud bucal parte integral del complejo cráneo-facial que participa en funciones fisiológicas indispensables para el ser humano, tales como alimentación, fonación, comunicación ${ }^{(2)}$. Un problema a nivel bucal puede limitar estas actividades cotidianas, generando molestias, dolor y deteriorando calidad de vida, pues en algún momento pueden generar deterioro a nivel mental reflejándose baja autoestima, tristeza y preocupación ${ }^{(3)}$. La CVRSB es un concepto que involucra no sólo el desarrollo de las actividades básicas con comodidad sino también la apreciación estética que hacen los individuos respecto a su boca durante las mismas; esto a su vez afecta autoestima, por lo cual, la apariencia de la sonrisa, el color y forma de los dientes, grosor y color de las encías y labios son considerados nuevos parámetros a tener en cuenta por los pacientes los cuales pueden cambiar su auto percepción e influir en su personalidad(4). Hertenstein et al., afirmaron que: "El comportamiento sonriendo en las fotografías es potencialmente indicativo, ya que este subyace disposiciones emocionales que tienen consecuencias directas e indirectas de vida" y probaron como esto traería consecuencias para la vida personal y hasta de pareja ${ }^{(5)}$. Son muchos los estudios que relacionan calidad de vida y salud bucal pero muestran asociaciones con presencia de caries dental, uso de prótesis dentales y presencia de restos radiculares en población adulta mayor ${ }^{(6)}$; sin embargo pocos estudios muestran asociaciones entre aspectos estéticos de la sonrisa y la calidad de vida. Por eso el objetivo del estudio es describir el impacto de satisfacción de la sonrisa sobre CVRSB de pacientes adultos de la Facultad de Odontología, Universidad de Cartagena, con el fin de aumentar evidencia científica y mejorar la calidad de la atención en salud bucal.

\section{MATERIAL Y MÉTODOS}

Estudio analítico corte transversal en 389 pacientes adultos que acuden por primera vez a la Facultad de Odontología de la Universidad de Cartagena, segundo semestre 2014 seleccionados por muestreo censal entre 18 y 81 años y que aceptaron participar previo consentimiento informado por escrito según requerimientos éticos ${ }^{(7)}$. Se aplicó cuestionario auto-suministrado para indagar: 1)Variables sociodemográficas: edad, sexo, procedencia, ingresos económicos, estado civil, servicios de salud, ocupación, nivel de escolaridad, 2) Valoración de la Sonrisa: Se evaluó clínicamente y se indagó por la satisfacción percibida por el paciente. Clínicamente implicó calibración de dos examinadores para valoración de dientes (tamaño, posición, forma y color), encías y labios que conforman la línea de la sonrisa según los criterios revisados por Passia et $a^{(8)}$. También se evaluó número de dientes, presencia de restos radiculares, restauraciones adaptadas y desadaptadas, dientes cariados y perdidos, presencia de cálculos dentales, uso y estado de prótesis dentales. Con respecto a la Satisfacción de la Sonrisa, se indagó a través de la escala Smile Perception Impact related Quality of Life (SPIRQoL) diseñada y validada por los autores en previa prueba piloto, la cual consta de 15 preguntas relacionadas con el impacto de la sonrisa sobre tres dominios: autoestima, función física y social; se calcula su puntaje mediante una sumatoria simple de respuestas tipo Likert, dando un rango entre 0 y 60 , donde el valor más alto indica mejor impacto de la sonrisa sobre la CVRSB; además se indagó por conocimientos sobre una sonrisa ideal y aspectos que cambiarían de su sonrisa, 3) CVRSB medida con el Indice General de salud oral relacionado con calidad de vida (GOHAI) el cual se encuentra validado(9) en poblaciones que cuentan con el idioma español, como México(10) y España(11), pero se realizó proceso de validación para la población colombiana, teniendo en cuenta las recomendaciones de realizar una nueva validación dadas las diferencias de contextos socioculturales y lingüísticas, tal y como lo recomiendan algunos autores ${ }^{(12,13)}$. Para esta escala se calcula su puntaje mediante una sumatoria simple de las respuestas, dando un rango entre 12 y 60 , donde el valor más alto indica mejor impacto de la salud bucal sobre la calidad de vida. Los datos fueron analizados utilizando STATA® 10.0 (Stata Corp, College Station, TX, EE.UU.).

Propiedades psicométricas de los instrumentos (validación)

En el proceso de validación de las escalas SPIRQoL y GOHAI, para determinar la validez de constructo (VC), se empleó análisis factorial confirmatorio (AFC): tres factores (Autoestima, función física y social) para SPIRQoL y tres factores para GOHAI (dolor, función física y función psicosocial). Posteriormente se obtuvieron índices de ajuste de estos modelos (cada escala): $x^{2}, p$-valor para $x^{2}$, grados de libertad, raíz cuadrada del error medio de aproximación (RCEMA) y su intervalo de confianza al $90 \%$, índice comparativo de ajuste (ICA) e índice de Tucker-Lewis (ITL). Empleando los criterios propuestos por Hu \& Bentler, se determinó como aceptable el ajuste de este modelo si: $p$-valor para $x 2>0,05$; RCEMA $\leq 0,06$; ICA $\geq 0,95$ e ITL $\geq 0,9514$. Finalmente, la consistencia interna para las dos escalas se estimó a través del coeficiente alfa de Cronbach ${ }^{(15)}$. El análisis factorial exploratorio para las escalas se realizó empleando Stata v.13.2 para Windows (StataCorp., College Station., TX., USA) y el análisis factorial confirmatorio empleando MPlus v.7.11 (Muthén \& Muthén, Los Angeles., CA., USA). También se realizó análisis descriptivo evaluado con medidas de tendencia central (medias y desviaciones estándar) del resultado total y dominios individuales del (SPIRQoL) y frecuencias y proporciones de los eventos de estudio, regresión de Poisson con varianza robusta para correlacionar variables sociodemográficas y la percepción de la sonrisa con la puntuación total del GOHAl y los dominios individuales $^{(16)}$, utilizando el conteo del resultado de CVRSB que ha sido desarrollado y utilizado ${ }^{(16)}$ (análisis uni y multivariado). Para los análisis categóricos (tablas 2 y 3 ) se usaron razones de tasas (RR) calculados con intervalos de confianza del $95 \%$.

\section{RESULTADOS}

El promedio de edad fue de 42,8 14,9 años, 56,8 \% sexo femenino, más de la mitad provienen del área urbana y pertenecen a estrato socioeconómico bajo, 46,7\% casados, 29,3\% han recibido educación hasta nivel de secundaria completa y un poco más de la mitad se halla trabajando, presentan bajos ingresos socioeconómicos y son beneficiarios de servicios públicos en salud. Con respecto al estado de salud bucal la mayoría conservan 19 dientes y 15,4 \% se realizó tratamientos ortodónticos o diseño de sonrisa (IC 95\%: 11,8-19). Para los resultados de las pruebas de concordancia entre los examinadores clínicos para la valoración clínica de la sonrisa, se realizó un análisis intra e inter-examinador, asumiendo un grado de acuerdo de 0,81 y 0,80 respectivamente, a través de la prueba Kappa Cohen. Con respecto a esta valoración, $72,7 \%$ y $65,8 \%$ presentan una alineación y color inadecuados de los dientes (IC 95\%: 68,3-77,1; IC 95\%:61-70,5). Al indagar por conocimientos sobre una sonrisa ideal, $31 \%$ consideran que esta corresponde a dientes grandes, blancos y sanos (IC 95\%: 26,2-35,4), seguidos de una dentadura sana; $2,8 \%$ la considera como una expresión facial que no requiere mostrar la dentadura (IC 95\%: 1,1-4,4). El aspecto de la sonrisa que muestran de primer interés para cambiar corresponde al tamaño y alineación de los dientes en un 29\% (IC 95\%: 24,5-33,5) seguidos del color en un 23,3\% (IC 95\%: 19,1-27,6) y 28,5\% refiere interés por someterse a un diseño de sonrisa (IC 95\%: 24-33). Al evaluar las propiedades psicométricas de las escalas SPIRQoL y GOHAI, los indicadores de ajuste obtenidos en el AFC (validez de constructo) fueron: para SPIRQoL: X2=143,55; grados de libertad: 59 ; $p$-valor para $X^{2}=0,00$; RCEMA=0,06; ICA=0,98 e ITL=0,98. Por su parte, la consistencia interna de la escala fue 0,89. Para GOHAI: $X^{2}=250,30$; grados de libertad: 65 ; p-valor para $x^{2}=0,00$; RCEMA $=0,13$; ICA $=0,91$ e ITL=0,89. Por su parte, la consistencia interna de la escala fue 0,87 .

Al evaluar el impacto de satisfacción de la sonrisa con respecto a la calidad de vida (SPIRQoL), 17,7\% (IC 95\%: 7,6-13,8) presentó insatisfacción de su sonrisa sobre la calidad de vida con mayor compromiso a nivel del dominio autoestima (Tabla 1). Al análisis univariado entre variables sociodemográficas y puntajes totales del SPIRQoL, se encontró que ser adulto joven presenta un impacto positivo sobre la satisfacción de la sonrisa mientras que contar con bajo nivel de escolaridad, ser de procedencia rural y presentar servicios de salud del sector público, muestra un impacto negativo (Tabla 2), al igual que todas las características clínicas evaluada de la sonrisa; en el modelo multivariado las variables que más mostraron un impacto negativo sobre la satisfacción de la sonrisa medido con SPIRQol fueron: procedencia rural, tamaño inadecuado de dientes, encías y labios inadecuados y presencia de caries dental (Tabla 2). Al realizar asociaciones de variables sociodemográficas, satisfacción de la sonrisa (SPIRQoL) y la valoración clínica de esta, con dominios individuales y totales del GOHAl también se mostraron asociaciones $(p \leq 0,05)$ (Tabla 3 ); en el modelo multivariado las variables que más mostraron un impacto negativo sobre la CVRSB medida con el GOHAl fueron: servicios de salud de carácter público y características inadecuadas al valorar clínicamente la sonrisa a excepción del color y posición de los dientes (Tabla 3).

\section{DISCUSIÓN}

Al interior de la consulta odontológica, estas escalas que miden 
Tabla 1. Impacto de Satisfacción de la Sonrisa relacionada con la Calidad de Vida (SPIRQoL) y sus dimensiones de referencia.

\begin{tabular}{|c|c|c|c|c|c|c|c|c|c|c|}
\hline \multirow{2}{*}{$\begin{array}{l}\text { Dimensiones } \\
\text { AUTOESTIMA }\end{array}$} & \multicolumn{2}{|c|}{ Nunca } & \multicolumn{2}{|c|}{ Casi Nunca } & \multicolumn{2}{|c|}{ Algunas veces } & \multicolumn{2}{|c|}{ Casi Siempre } & \multicolumn{2}{|c|}{ Siempre } \\
\hline & Frecuencia & $\%$ & Frecuencia & $\%$ & Frecuencia & $\%$ & Frecuencia & $\%$ & Frecuencia & $\%$ \\
\hline $\begin{array}{l}\text { ¿La sonrisa que tiene actualmente le } \\
\text { confiere satisfacción y seguridad? }\end{array}$ & 124 & 31,8 & 105 & 26,9 & 83 & 21,3 & 39 & 10,0 & 38 & 9,7 \\
\hline $\begin{array}{c}\text { ¿Alguna vez ha sentido vergüenza por } \\
\text { su sonrisa? }\end{array}$ & 102 & 26.2 & 59 & 15.1 & 113 & 29,0 & 77 & 19,7 & 38 & 9,7 \\
\hline $\begin{array}{c}\text { ¿Se ha sentido cohibido al sonreir } \\
\text { porque le desagrada el color de sus } \\
\text { dientes? }\end{array}$ & 134 & 34,4 & 77 & 19,7 & 90 & 23,1 & 70 & 17,9 & 18 & 4,6 \\
\hline $\begin{array}{l}\text { ¿Ha sentido que los demás lo } \\
\text { observan mal porque su sonrisa no es } \\
\text { agradable? }\end{array}$ & 167 & 42,9 & 76 & 19,5 & 92 & 23,6 & 43 & 11,0 & 11 & 2,83 \\
\hline $\begin{array}{l}\text { ¿Piensa que las sonrisas de las otras } \\
\text { personas son mucho mejores que la } \\
\text { suya? }\end{array}$ & 63 & 16,2 & 54 & 13,8 & 120 & 30,8 & 78 & 20,0 & 74 & 19,0 \\
\hline \multicolumn{11}{|l|}{ FUNCION FÍSICA } \\
\hline $\begin{array}{c}\text { ¿Alguna mal posición de sus dientes le } \\
\text { ha generado un obstáculo físico para } \\
\text { sonreír? }\end{array}$ & 209 & 53,7 & 57 & 14,6 & 75 & 19,2 & 26 & 6,6 & 22 & 5,6 \\
\hline $\begin{array}{c}\text { ¿Esta conforme con la forma y tamaño } \\
\text { de sus labios y encias? }\end{array}$ & 133 & 34 & 75 & 19,2 & 38 & 9,7 & 62 & 15,9 & 81 & 20,7 \\
\hline $\begin{array}{l}\text { ¿En ocaciones evita sonreir porque el } \\
\text { tamaño de sus dientes es inadecuado? }\end{array}$ & 155 & 39,8 & 58 & 14,9 & 77 & 19,9 & 63 & 16,2 & 36 & 9,25 \\
\hline $\begin{array}{c}\text { ¿Se le dificultado la pronunciacion de } \\
\text { algunas palabras por malposición de } \\
\text { sus dientes? }\end{array}$ & 259 & 66,5 & 38 & 9,7 & 59 & 15,1 & 24 & 6,1 & 9 & 2,31 \\
\hline $\begin{array}{l}\text { Has presentado dolor en tus dientes o } \\
\text { encías al sonreir? }\end{array}$ & 308 & 79,1 & 28 & 7,20 & 39 & 10,0 & 13 & 3,34 & 1 & 0,26 \\
\hline \multicolumn{11}{|l|}{ SOCIAL } \\
\hline $\begin{array}{c}\text { ¿Se ha sentido ofendido o } \\
\text { discriminado por la apariencia de su } \\
\text { sonrisa? }\end{array}$ & 202 & 51,9 & 72 & 18,5 & 72 & 18,5 & 33 & 8,48 & 10 & 2,57 \\
\hline $\begin{array}{l}\text { ¿Ha adoptado posturas o hábitos para } \\
\text { esconder su sonrisa? }\end{array}$ & 147 & 37,7 & 45 & 11,5 & 78 & 20,5 & 60 & 15,4 & 59 & 15,1 \\
\hline $\begin{array}{c}\text { ¿Ha sentido que por su sonrisa se } \\
\text { le han limitado las oportunidades } \\
\text { laborales? }\end{array}$ & 265 & 68,1 & 36 & 9,25 & 57 & 14,6 & 15 & 3,86 & 16 & 4,1 \\
\hline $\begin{array}{l}\text { ¿Ha sentido que por su sonrisa } \\
\text { se han limitado sus relaciones } \\
\text { interpersonales? }\end{array}$ & 287 & 73,7 & 40 & 10,2 & 44 & 11,3 & 10 & 2,5 & 8 & 2,0 \\
\hline $\begin{array}{c}\text { ¿Su sonrisa ha sido una limitante en } \\
\text { su vida amorosa? }\end{array}$ & 299 & 76,8 & 31 & 7,9 & 39 & 10,0 & 12 & 3,0 & 8 & 2,0 \\
\hline
\end{tabular}

CVRSB (GOHAI) y el Smile Perception Impact related Quality of Life (SPIRQoL), diseñada esta última por los autores, evalúan percepciones y expectativas de los pacientes frente a los tratamientos estéticos y odontológicos a realizar, teniendo en cuenta que se puede presentar una equivocada autopercepción de estética. Algunas limitaciones del estudio se dan por estas percepciones emitidas por los pacientes, por lo cual estos resultados deben ser analizados teniendo en cuenta la probabilidad del sesgo subsecuente; sin embargo, constituyen un aporte académico al entendimiento de la multi-dimensionalidad de la enfermedad bucal.

Este estudio muestra una asociación entre la insatisfacción con la sonrisa y su impacto negativo sobre la calidad de vida, al emplear el SPIRQoL, comprometiendo principalmente el dominio autoestima, además del dominio función y aspecto social del individuo, coincidiendo con autores como Goldstein y Lancaster en Estados Unidos ${ }^{(17)}$. Actualmente el atractivo facial se encuentra íntimamente relacionado con el atractivo de la sonrisa, una de las más importantes expresiones faciales, al momento de demostrar simpatía, aprobación y aprecio ${ }^{(18)}$. Incluso a nivel social el atractivo facial influye en el éxito de atracción al sexo opuesto, oportunidades de relacionarse, evaluaciones de personalidad, rendimiento y las perspectivas de empleo(19). Algunos pacientes se encontraron inconformes con el tamaño de sus dientes, forma y tamaño de labios y encías e impactó de manera negativa sobre CVRSB al ser evaluado con el GOHAl. De hecho un diseño de sonrisa requiere la integración entre la composición facial y la composición dental, en la cual la composición facial considera los tejidos duros y blandos de la cara, en tanto que la composición dental incluye específicamente a los dientes y la relación de estos con los tejidos gingivales ${ }^{(20)}$. Al respecto, Van der Geld en 2007, destaca la importancia de considerar en la estética de la sonrisa, no solo componentes dentales como posición, tamaño, forma y color a nivel dental, sino también elementos del aspecto gingival y contorno de los labios, pues estos últimos serán el marco de todos los componentes, formando un complejo armónico y simétrico ${ }^{(4)}$. Autores como Shah en 2014(21), reportan que el color y el grosor gingival excesivo así como la existencia de un mal estado de salud periodontal afectan negativamente los juicios individuales sobre el atractivo de la sonrisa, así como la estimación de la confianza en sí mismo, amabilidad, honradez e inteligencia. Estos hallazgos deberían ser considerados a la hora de realizar un diseño de sonrisa o tratamientos estéticos bucales. En relación a la valoración clínica de la sonrisa de manera conjunta, se ha expuesto que se encuentra determinada por la coincidencia entre las líneas medias dentales y faciales, las proporciones dentales, estética gingival, color de los dientes e inclinación del plano oclusal(22); sin embargo en el estudio el diagnóstico clínico se limitó a aspectos relacionados con dentición, tejidos gingivales y labios, olvidando mediciones faciales externas que podrían repercutir en la percepción estética de los pacientes, por lo cual se sugieren que se incluyan en próximas investigaciones. Con respecto a lo que se considera una sonrisa ideal por los pacientes del estudio, cerca del $31 \%$ se refirió a dientes grandes, blancos y sanos, coincidiendo con 
Tabla 2. Análisis Univariado y Multivariado ajustado por variables sociodemográficas y condiciones clínicas bucales asociadas con el puntaje total del Smile Perception Impact related Quality of Life (SPIRQoL).

\begin{tabular}{|c|c|c|c|c|}
\hline \multirow{2}{*}{$\begin{array}{c}\text { Variables } \\
\text { independientes }\end{array}$} & \multicolumn{2}{|c|}{$\begin{array}{l}\text { Análisis univariado } \\
\text { Puntaje Total }\end{array}$} & \multicolumn{2}{|c|}{$\begin{array}{c}\text { Análisis multivariado } \\
\text { Puntaje Total }\end{array}$} \\
\hline & RR (IC: 95\%) & $\mathrm{p}$ & RR (IC: $95 \%)$ & $\mathrm{p}$ \\
\hline \multicolumn{5}{|l|}{ Edad } \\
\hline Jóvenes & $0,37(0,20-0,76)$ & 0,001 & $\dagger$ & \\
\hline \multicolumn{5}{|l|}{ Adultos Maduros } \\
\hline \multicolumn{5}{|l|}{ Sexo } \\
\hline \multicolumn{5}{|l|}{ Masculino } \\
\hline Femenino & $0,82(0,48-1,42)$ & 0,5 & & \\
\hline \multicolumn{5}{|l|}{ Estado Civil } \\
\hline Solteros & $0,57(0,31-1,04)$ & 0,06 & $\dagger$ & \\
\hline \multicolumn{5}{|l|}{ Casados } \\
\hline \multicolumn{5}{|l|}{ Nivel de Escolaridad } \\
\hline \multicolumn{5}{|l|}{ Alto Nivel } \\
\hline Bajo Nivel & $2,9(1,74-5,1)$ & 0,000 & $\dagger$ & \\
\hline \multicolumn{5}{|l|}{ Trabajan? } \\
\hline No & $1,8(1,03-3,15)$ & 0,038 & $\dagger$ & \\
\hline \multicolumn{5}{|l|}{$\mathrm{Si}$} \\
\hline \multicolumn{5}{|l|}{ Procedencia } \\
\hline Rural & $2,6(1,5-4,43)$ & 0,000 & $2,27(1,4-3,6)$ & 0,001 \\
\hline \multicolumn{5}{|l|}{ Urbana } \\
\hline \multicolumn{5}{|l|}{ Seguridad Social } \\
\hline \multicolumn{5}{|l|}{ Sector Privado } \\
\hline Sector Publico & $2,07(1,14-3,76)$ & 0,017 & $\dagger$ & \\
\hline \multicolumn{5}{|l|}{ Estrato Socioeconómico } \\
\hline \multicolumn{5}{|l|}{ Alto Estrato } \\
\hline Bajo Estrato & $1,5(0,74-3,2)$ & 0,24 & & \\
\hline \multicolumn{5}{|l|}{ Ingresos Socioeconómicos } \\
\hline Más de 1 SMMLV & $1,27(0,72-2,2)$ & 0,4 & & \\
\hline \multicolumn{5}{|l|}{ Menos de 1 SMMLV } \\
\hline \multicolumn{5}{|l|}{ Valoración Clínica de la Sonrisa } \\
\hline Tamaño de dientes inadecuados & $9,13(3,6-22,6)$ & 0,000 & $10(3,12-32,1)$ & 0,000 \\
\hline Color de dientes inadecuados & $1,4(0,78-2,74)$ & 0,25 & & \\
\hline Posición de dientes inadecuada & $3,07(1,24-7,57)$ & 0,015 & $\dagger$ & \\
\hline Encías inadecuadas & $4,02(2,2-7,28)$ & 0,000 & $2,4(1,3-4,3)$ & 0,004 \\
\hline Labios inadecuados & $4,38(2,53-7,59)$ & 0,000 & $2,5(1,5-4,4)$ & 0,001 \\
\hline Menos de 19 dientes & $2,33(1,29-4,2)$ & 0,005 & $\dagger$ & \\
\hline Caries Dental & $3,8(1,6-8,7)$ & 0,002 & $2,8(1,36-5,9)$ & 0,005 \\
\hline Restos Radiculares & $3,08(1,9-4,9)$ & 0,000 & $\dagger$ & \\
\hline Sonrisa Clínicamente Inadecuada & $2,6(0,66-10)$ & 0,16 & $0,14(0,025-0,83$ & 0,03 \\
\hline
\end{tabular}

*Valor del modelo: 86,31; valor de la p: 0,000, +Variables no asociadas con el respectivo dominio al final del modelo multivariado después del ajuste.

Kershaw et al., ${ }^{(23)}$ quien reporta que los pacientes asocian el poseer dientes oscuros con la presencia de caries dental y una pobre higiene bucal. Esta percepción de idealidad podría estar relacionada con el contexto social actual, impulsado principalmente por los medios de comunicación, quienes difunden el concepto de estética dental con el poseer perfiles más calificados y confiables, de tal forma que el cumplimiento de estas condiciones va a influir en un fenómeno psicológico que implica respuestas neurológicas positivas o negativas en las relaciones interpersonales y en el grado de autoestima y autoimagen ${ }^{(24)}$. El contar con servicios de salud de carácter público fue otra variable que impactó de forma negativa sobre la CVRSB además de pertenecer a estratos socioeconómicos bajos. Quizás existen diferencias en la atención odontológica brindada entre servicios de carácter público y privado; Brennan en 2008 en Australia(25), $^{(25}$ reporta que existe un contraste entre pacientes que reciben servicios odontológicos de instituciones públicas y privadas, puesto que para el caso del sector público, se evidencia un problema de escasez de recursos y otros factores como prolongados períodos de espera para atención, que de manera sinérgica favorecen el curso negativo de las condiciones de salud bucal iniciales por las cuales se consulta, pues estas empeoran a tal grado que cuando por fin son atendidas, la asistencia de emergencia suele ser el camino de elección y este manejo se traduce en un mayor porcentaje de procedimientos tipo extracciones, un menor porcentaje de servicios preventivos y de mantenimiento y un deterioro de la calidad de vida, situación muy parecida a la sentida en la población colombiana. En este mismo sentido, los sujetos estudiados que proceden de zonas rurales muestran un impacto negativo sobre la CVRSB, hallazgos similares a 
Tabla 3. Análisis Univariado y Multivariado ajustado por variables sociodemográficas y condiciones clínicas bucales asociadas con el puntaje total del General Oral Health Assesment Index (GOHAI).

\begin{tabular}{|c|c|c|c|c|c|c|c|c|c|c|}
\hline \multirow{3}{*}{\begin{tabular}{|c|}
$\begin{array}{c}\text { Variables } \\
\text { independientes }\end{array}$ \\
$\begin{array}{c}\text { Condiciones } \\
\text { sociodemográficas }\end{array}$ \\
\end{tabular}} & \multicolumn{6}{|c|}{ Sección de impacto o dominios del GOHAI } & \multirow{2}{*}{\multicolumn{2}{|c|}{$\begin{array}{c}\begin{array}{c}\text { GOHAl Análisis } \\
\text { univariado }\end{array} \\
\text { Puntaje total }\end{array}$}} & \multirow{2}{*}{\multicolumn{2}{|c|}{$\begin{array}{c}\begin{array}{c}\text { GOHAl *Análisis } \\
\text { multivariado }\end{array} \\
\text { Puntaje Total } \\
\end{array}$}} \\
\hline & \multicolumn{2}{|l|}{ Física } & \multicolumn{2}{|c|}{ Sicosocial } & \multicolumn{2}{|l|}{ Dolor } & & & & \\
\hline & RR (95\% IC) & $\mathbf{p}$ & RR (95\% IC) & $\mathbf{p}$ & RR (95\% IC) & $\mathbf{p}$ & $\operatorname{RR}(95 \%$ IC) & $\mathbf{p}$ & RR $(95 \%$ IC) & $\mathbf{P}$ \\
\hline \multicolumn{11}{|l|}{ Edad } \\
\hline Jóvenes & $0,92(0,87-0,98)$ & 0,01 & $0,87(0,79-0,95)$ & 0,003 & $0,81(0,69-0,96)$ & 0,01 & $0,88(0,83-0,94)$ & 0,000 & $\dagger$ & \\
\hline \multicolumn{11}{|l|}{ Adultos Maduros } \\
\hline \multicolumn{11}{|l|}{ Sexo } \\
\hline \multicolumn{11}{|l|}{ Masculino } \\
\hline Femenino & $0,95(0,90-1,01)$ & 0,13 & $0,92(0,84-1,01)$ & 0,09 & $0,91(0,77-1,07)$ & 0,2 & $0,93(0,87-1)$ & 0,05 & & \\
\hline \multicolumn{11}{|l|}{ Estado Civil } \\
\hline Solteros & $1,02(0,96-1,08)$ & 0,4 & $0,93(0,84-1,02)$ & 0,15 & $0,92(0,78-1,09)$ & 0,3 & $0,96(0,90-1,03)$ & 0,3 & & \\
\hline \multicolumn{11}{|l|}{ Casados } \\
\hline \multicolumn{11}{|c|}{ Nivel de Escolaridad } \\
\hline \multicolumn{11}{|c|}{ Alto Nivel } \\
\hline Bajo Nivel & $1,08(1,02-1,15)$ & 0,008 & $1,28(1,16-1,4)$ & 0,000 & $1,3(1,14-1,6)$ & 0,000 & $1,2(1,12-1,3)$ & 0,000 & $\dagger$ & \\
\hline \multicolumn{11}{|l|}{ Trabajan? } \\
\hline No & $0,98(0,92-1,03)$ & 0,5 & $0,96(0,88-1,06)$ & 0,5 & $0,89(0,75-1,05)$ & 0,1 & $0,96(0,90-1,03)$ & 0,29 & & \\
\hline \multicolumn{11}{|l|}{$\mathrm{Si}$} \\
\hline \multicolumn{11}{|l|}{ Procedencia } \\
\hline Rural & $0,98(0,92-1,04)$ & 0,6 & $1,10(0,99-1,22)$ & 0,06 & $1,28(1,08-1,5)$ & 0,004 & $1,07(0,99-1,15)$ & 0,059 & & \\
\hline \multicolumn{11}{|l|}{ Urbana } \\
\hline \multicolumn{11}{|l|}{ Seguridad Social } \\
\hline \multicolumn{11}{|l|}{ Sector Privado } \\
\hline Sector Público & $1,11(1,05-1,17)$ & 0,000 & $1,3(1,2-1,4)$ & 0,000 & $1,26(1,07-1,5)$ & 0,005 & $1,21(1,13-1,3)$ & 0,000 & $1,08(1,02-1,15)$ & 0,004 \\
\hline \multicolumn{11}{|c|}{ Estrato Socioeconómico } \\
\hline \multicolumn{11}{|c|}{ Alto Estrato } \\
\hline Bajo Estrato & $1,01(0,95-1,08)$ & 0,54 & $1,2(1,08-1,35)$ & 0,001 & $1,3(1,12-1,7)$ & 0,002 & $1,14(1,05-1,23)$ & 0,001 & $\dagger$ & \\
\hline Ingresos Socioeco & nómicos & & & & & & & & & \\
\hline Más de 1 SMMLV & & & & & & & & & & \\
\hline $\begin{array}{l}\text { Menos de } 1 \\
\text { SMMLV }\end{array}$ & $0,94(0,89-0,99)$ & 0,048 & $1,10(1-1,21)$ & 0,03 & $1,3(1,09-1,5)$ & 0,002 & $1,05(0,98-1,12)$ & 0,1 & $\dagger$ & \\
\hline $\begin{array}{l}\text { Insatisfacción } \\
\text { con la Sonrisa } \\
\text { (SPIRQoL) }\end{array}$ & $1,04(0,95-1,1)$ & 0,3 & $1,6(1,6-1,8)$ & 0,000 & $1,6(1,37-1,9)$ & 0,000 & $1,41(1,3-1,5)$ & 0,000 & $1,19(1,2-1,4)$ & 0,000 \\
\hline Valoración Clínica d & le la Sonrisa & & & & & & & & & \\
\hline $\begin{array}{l}\text { Tamaño de } \\
\text { los dientes } \\
\text { inadecuados }\end{array}$ & $1,13(1,07-1,2)$ & 0,000 & $1,4(1,3-1,6)$ & 0,000 & $1,6(1,4-1,9)$ & 0,000 & $1,3(1,24-1,40)$ & 0,000 & $1,14(1,06-1,2)$ & 0,000 \\
\hline $\begin{array}{l}\text { Color de } \\
\text { los dientes } \\
\text { inadecuados }\end{array}$ & $1,06(1,0-1,13)$ & 0,04 & $1,2(1,06-1,3)$ & 0,003 & $1,29(1,06-1,5)$ & 0,008 & $1,14(1,05-1,23)$ & 0,001 & $\dagger$ & \\
\hline $\begin{array}{l}\text { Posición de } \\
\text { los dientes } \\
\text { inadecuadas }\end{array}$ & $1,10(1,03-1,18)$ & 0,002 & $1,4(1,2-1,6)$ & 0,000 & $1,5(1,2-1,8)$ & 0,000 & $1,27(1,16-1,36)$ & 0,000 & $\dagger$ & \\
\hline $\begin{array}{l}\text { Encías } \\
\text { inadecuadas }\end{array}$ & $1,03(0,97-1,09)$ & 0,23 & $1,4(1,2-1,5)$ & 0,000 & $1,5(1,3-1,8)$ & 0,000 & $1,24(1,16-1,32)$ & 0,000 & $1,09(1,03-1,16)$ & 0,003 \\
\hline $\begin{array}{l}\text { Labios } \\
\text { inadecuados }\end{array}$ & $1,02(0,91-1,14)$ & 0,6 & $1,4(1,2-1,6)$ & 0,000 & $0,95(0,7-1,2)$ & 0,74 & $1,18(1,05-1,32)$ & 0,003 & $\dagger$ & \\
\hline $\begin{array}{l}\text { Menos de } 19 \\
\text { dientes }\end{array}$ & $1,1(1,01-1,2)$ & 0,02 & $1,24(1,11-1,4)$ & 0,000 & $1,5(1,24-1,8)$ & 0,000 & $1,21(1,11-1,33)$ & 0,000 & $1,10(1,02-1,2)$ & 0,009 \\
\hline Caries Dental & $1,04(0,97-1,10)$ & 0,2 & $1,3(1,2-1,4)$ & 0,000 & $1,46(1,2-1,74)$ & 0,000 & $1,20(1,12-1,29)$ & 0,000 & $1,1(1,04-1,2)$ & 0,001 \\
\hline $\begin{array}{l}\text { Restos } \\
\text { Radiculares }\end{array}$ & $1,06(0,9-1,14)$ & 0,07 & $1,29(1,2-1,4)$ & 0,000 & $1,6(1,4-1,9)$ & 0,000 & $1,24(1,16-1,32)$ & 0,000 & $\dagger$ & \\
\hline $\begin{array}{l}\text { Sonrisa } \\
\text { Clínicamente } \\
\text { Inadecuada }\end{array}$ & $1,19(1,08-1,3)$ & 0,000 & $1,9(1,6-2,3)$ & 0,000 & $2,9(1,98-4,23)$ & 0,000 & $1,59(1,43-1,76)$ & 0,000 & $1,3(1,17-1,45)$ & 0,000 \\
\hline
\end{tabular}

82 | Rev. Clin. Periodoncia Implantol. Rehabil. Oral Vol. 11(2); 78-83, 2018. 
los de Sanadhya et al., en $2015^{(26)}$, quien reportó que la condición de salud bucal fue inferior en los sujetos de procedencia rural y estrato socioeconómico bajo, lo cual podría estar relacionado con ausencia de higiene bucal y representar un factor de riesgo para el incremento de enfermedades bucales. Se podría también sugerir, que este impacto negativo obedece a limitaciones en el acceso a servicios odontológicos en estas zonas; en zonas urbanas existe mejor cobertura en servicios de salud bucal por la presencia de un mayor número de recurso humano (Odontólogos), de escuelas de odontología y hospitales. Cabe resaltar que en Colombia el gremio odontológico se concentra mucho más en las ciudades por la inseguridad y problemas de orden político y social vividos en zonas rurales, lo que a su vez dificulta la llegada a estos lugares de servicios odontológicos diferentes a los servicios generales, tales como la Ortodoncia y Rehabilitación Oral y Estética que pudieran mejorar el estado de salud bucal de la población no solo en relación a función, sino en relación al aspecto estético de su sonrisa. A esto se puede agregar que es posible que en estas áreas rurales se dé por tanto más prioridad a la atención de otros eventos de salud que a la salud bucal no considerada elemento importante para el mejoramiento de la calidad de vida.

\section{CONCLUSIONES}

La insatisfacción de la sonrisa y la apariencia clínica inadecuada de dientes, encías y labios impactan de manera negativa sobre la calidad de vida de pacientes adultos, especialmente en aquellos de procedencia rural y con servicios de salud de carácter público.

\section{CONFLICTO DE INTERÉS.}

Los autores declaramos que no existe conflicto de interés con la institución que pudiera influir en los hallazgos observados.

Institución centro de trabajo. Universidad de Cartagena, Colombia.

\section{AGRADECIMIENTOS}

A Lauren Manjarrez y Clara Elena Robles en la elaboración y diseño de la escala SPIRQoL y al Dr. Miguel Simancas Pallares, Magister en Epidemiología Clínica y docente de la Facultad de Odontología de la Universidad de Cartagena por el proceso de validación del SPIRQoL y GOHAI.

\section{RESPONSABILIDADES ÉTICAS}

Protección de personas y animales. Los autores declaran que los procedimientos seguidos se conformaron a las normas éticas del comité de experimentación humana responsable y de acuerdo con la Asociación Médica Mundial y la Declaración de Helsinki.

Confidencialidad de los datos. Los autores declaran que han seguido los protocolos de su centro de trabajo sobre la publicación de datos de pacientes.

Derecho a la privacidad y consentimiento informado. Los autores han obtenido el consentimiento informado de los pacientes y/o sujetos referidos en el artículo. Este documento obra en poder del autor de correspondencia.

\section{Bibliografía}

1. Sischo I, Broder HL. Oral health-related quality of life what, why, how, and future implications. J Dent Res. 2011;90(11):1264-70.

2. Bennadi D, Reddy CVK. Oral health related quality of life. J Int Soc Prev Community Dent. 2013; 3(1):1-6.

3. Misrachi Launert CM, Espinoza Santander I. Utilidad de las mediciones de la calidad de vida relacionada con la salud. Rev Dent Chile. 2005;96(2):28-35.

4. Van der Geld P, Oosterveld P, Van Heck G, Kuijpers-Jagtman AM. Smile attractiveness: self-perception and influence on personality. Angle Orthod 2007;77(5):759-65

5. Hertenstein MJ, Hansel CA, Butts AM, Hile SN. Smile intensity in photographs predicts divorce later in life. Motiv Emot. 2009;33(2):99-105.

6 . Duque-Duque VE, Tamayo-Castrillón J, Echeverri-Cadavid PA, Gutiérrez-Osorio AY, Sepúlveda-Correa D, Giraldo-Ramírez O, et al. Calidad de vida relacionada con la salud bucal en adultos mayores que consultan a la IPS Universitaria de Medellín y sus factores asociados. CES Odontol. 2013;26(1):10-23.

7. Ministerio de Salud. Resolución Número 8430 Colombia 1993

8. Passia N, Blatz M, Strub JR. Is the smile line a valid parameter for esthetic evaluation? A systematic literature review. Eur J Esthet Dent. 2011 Autumn;6(3):314-27.

9. Atchison KA, Dolan TA. Development of the geriatric oral health assessment index. J Dent Educ. 1990;54(11):680-7.

10. Sánchez S, Heredia E, Juárez T, Gallegos K, Espinel C, De la Fuente J, García C. Psychometric properties of the General Oral Health Assessment Index (GOHAI) and dental status of an elderly Mexican population. J Public Health Dent 2010;70(4):300-7.

11. Pinzón-Pulido SA, Gil-Montoya JA.Validación del índice de valoración de salud oral en geriatría en una población geriátrica institucionalizada de Granada. Rev Esp Geriatr Gerontol. 1999;34:273-82.

12. Sánchez r, Echeverry J. Validación de escalas de medición en salud. Rev. Salud Pública. 2004;6(3):302-318.

13. Hu LT, Bentler PM. Cutoff criteria for fit indexes in covariance structure analysis: Conventional criteria versus new alternatives. Structural Equation Modeling. 1999;6(1):1-55
14. Oviedo $\mathrm{H}$, Campo Arias A. Aproximación al uso del coeficiente alfa de Cronbach. Rev Col Psiquiatr. 2005;34(4):572-580.

15. Biazevic MG, Antunes JL, Togni J, de Andrade FP, de Carvalho MB, WünschFilho V. Immediate impact of primary surgery on health related quality of life of hospitalized patients with oral and oropharyngeal cancer. J Oral Maxillofac Surg. 2008:66:1343-50

16. Goldstein RE, Lancaster JS. Survey of patient attitudes toward current esthetic procedures. J Prosthet Dent. 1984;52(6):775-80.

17. Cao L, Zhang K, Bai D, Jing Y, Guo Y. Effect of maxillary incisor labiolingual inclination and anteroposterior position on smiling profile esthetics. Angle Orthod. 2011;81(1):121-29

18. Anthony C. Little, Benedict C. Jones, Lisa M. DeBruine. Facial attractiveness: evolutionary based research. Philos Trans R Soc Lond B Biol Sci. 2011;12; 366(1571):1638-1659.

19. Bhuvaneswaran M. Principles of smile design. J Conserv Dent. 2010;13(4):224 32.

20.Shah R, Shah S. Oral rehabilitation of a patient with ectodermal dysplasia: A multidisciplinary approach. J Nat Sci Biol Med. 2014 Jul;5(2):462-6.

21. Cotrim ER, Vasconcelos Júnior ÁV, Haddad ACSS, Reis SAB. Perception of adults' smile esthetics among orthodontists, clinicians and laypeople. Dental Press J Orthod. 2015; 20(1):40-4

22. Kershaw S, Newton JT, Williams DM. The influence of tooth colour on the perceptions of personal characteristics among female dental patients: comparisons of unmodified, decayed and whitened teeth. Br Dent J. 2008;204(5):E9-E.

23. Furnham A. Sex, IQ, and emotional intelligence. Psychol Rep. 2009;105(3 Pt 2):1092-4

24. Brennan DS, Luzzi L, Roberts-Thomson KF. Dental service patterns among private and public adult patients in Australia. BMC Health Serv Res. 2008;3;8:1. 25. Sanadhya S, Aapaliya P, Jain S, Sharma N, Choudhary G, Dobaria N. Assessment and comparison of clinical dental status and its impact on oral health-related quality of life among rural and urban adults of Udaipur, India: A cross-sectional study. J Basic Clin Pharm. 2015;6(2):50. 